\title{
Stellar activity cycles in a model for magnetic flux generation and transport
}

\author{
Emre Işık \\ Department of Physics, Faculty of Science \& Letters, Istanbul Kültür University \\ 34156, Bakırköy, Istanbul, Turkey \\ email: e.isik@iku.edu.tr
}

\begin{abstract}
We present results from a model for magnetic flux generation and transport in cool stars and a qualitative comparison of models with observations. The method combines an $\alpha \Omega$-type dynamo at the base of the convection zone, buoyant rise of magnetic flux tubes, and a surface flux transport model. Based on a reference model for the Sun, numerical simulations were carried out for model convection zones of G- and K-type main sequence and subgiant stars. We investigate magnetic cycle properties for stars with different rotation periods, convection zone depths, and dynamo strengths. For a Sun-like star with $P_{\text {rot }}=9 \mathrm{~d}$, we find that a cyclic dynamo can underly an apparently non-cyclic, 'flat' surface activity, as observed in some stars. For a subgiant K1 star with $P_{\text {rot }}=2.8 \mathrm{~d}$ the long-term activity variations resemble the multi-periodic cycles observed in V711 Tau, owing to high-latitude flux emergence, weak transport effects and stochastic processes of flux emergence.
\end{abstract}

Keywords. stars: activity, stars: interiors, stars: magnetic fields, (magnetohydrodynamics:) MHD, Sun: interior, Sun: magnetic fields

\section{Introduction}

Magnetically active stars are important to better understand the limits and the behaviour of the solar dynamo, in addition to how it used to operate during the early stages of solar evolution. The level of activity increases with the rotation rate and the fractional depth of the convection zone, up to a saturation level for ultra-fast rotators and fully convective stars. The distribution and coverage of stellar magnetic regions differ significantly from the solar patterns as the Rossby number (ratio of the rotation period to convective turn-over time) decreases (Strassmeier 2009). Temporal properties of magnetic activity are also of interest when comparing the solar cycle with stellar cycles. Multi-periodic stellar cycles have also been reported (Oláh et al. 2009). We present a model for the generation and transport of stellar magnetic fields (Işık, Schmitt \& Schüssler 2007, 2011) and discuss possible magnetic flux transport mechanisms in active stars, in comparison with observations of stellar activity cycles.

\section{The model}

We have recently developed a three-part model for solar and stellar magnetic flux generation and transport (Işık, Schmitt \& Schüssler 2007, 2011). In the first part, a onedimensional mean-field $\alpha \Omega$ dynamo with a saturation mechanism related to buoyant flux removal is set up at the position of the overshoot region at the base of the convection zone (Schmitt \& Schüssler 1989). For faster rotators, the strength of the $\alpha$-effect is assumed to scale with the rotation rate. This is similar to the assumption that the differential rotation rate scales with the activity level and thus with the rotation rate, as suggested recently by Saar (2011), based on observations of a homogeneous sample of cool stars. In the 


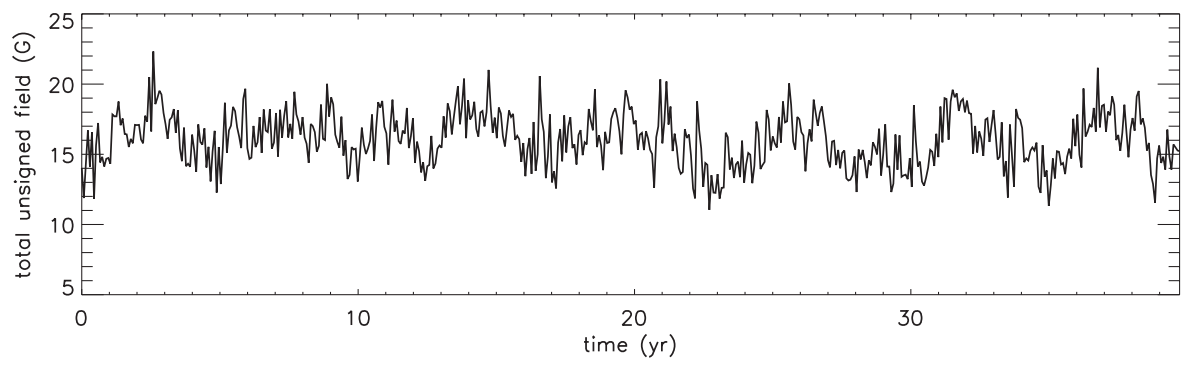

Figure 1. Variation of total unsigned magnetic flux density for the Sun-like model with $P_{\text {rot }}=9 \mathrm{~d}$. The right axis shows the corresponding field strength.

second part, time-latitude distribution of the dynamo-generated toroidal field determines the probability distribution that a flux tube would become unstable at a given latitude and time. The tubes are assumed to have properties given by the criteria for magnetic buoyancy instability at a given latitude. The emergence latitudes and tilt angles of BMRs are determined by numerical simulations of flux tube rise through the convection zone. These results are used as input for the flux transport model at the surface (the third part), including effects of differential rotation, meridional flow, horizontal and radial diffusion. The number of emerging BMRs (per activity cycle) is scaled up with the rotation rate and their areas are determined randomly, following a probability distribution set by the Sun-like area distribution, $N(A) \propto A^{-2}$. The details of the model construction are described by Işık, Schmitt \& Schüssler (2011).

\section{Sun-like stars: disappearance of cycles}

For the Sun-like models, the cycle period decreases with the rotation rate, owing to stronger $\alpha$-effect. For $P_{\text {rot }} \gtrsim 10 \mathrm{~d}$, the polar fields become stronger with increasing rotation rate, owing to increasing frequency of flux emergence and tilt angles, until another effect starts to set the stage: the increasing dynamo strength (the $\alpha$-effect), thus the increasing cycle frequency. For $P_{\text {rot }} \lesssim 10 \mathrm{~d}$ polar regions undergo field reversals with increasingly high frequencies, so that the peak polar fields do not reach the field strengths expected from the emerging magnetic flux. For most of these cases the magnetic flux variations show clear cycles.

For $P_{\text {rot }}=9 \mathrm{~d}$, the level of total magnetic flux is considerably higher than the solar values, but the underlying periodic dynamo cycle is 'obscured' by the combined effects of rise and surface transport of magnetic flux (Fig. 1). The reason for the 'invisible' stellar cycle is that during 'magnetic minima' the magnetic flux at high latitudes become comparable to that of low latitudes during activity maxima. This intermediate rotator case represents a moderately active, but non-cyclic (or weakly cyclic) configuration (in fact, there is a weak cycle with $\sim 6$-yr period). The model indicates a possibility that such stars may not be in a Maunder minimum state and are still observed as non-cyclic. Observational evidence on the existence of such stars already supports this possibility (Hall \& Lockwood 2004).

\section{K stars: from regular to fluctuating cycles}

Işı, Schmitt \& Schüssler (2011) have applied the Sun-like model to K-type stars with different radii. The first case is a K0-type main-sequence star, rotating 13 times faster than the Sun. Similar to the Sun-like star with the same rotation rate, large tilt 


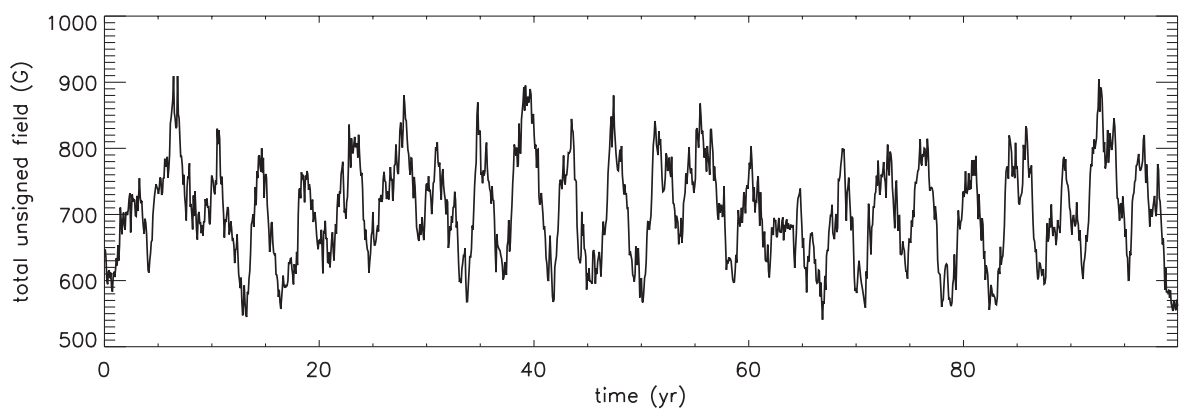

Figure 2. Variation of the surface-integrated unsigned magnetic field strength for the K1IV-type fast rotator with $P_{\text {rot }}=2.8 \mathrm{~d}$.

angles lead to formation of activity belts, but in this case as shifted towards the poles, because of an additional geometric effect. Buoyant flux tubes experience a substantial Coriolis acceleration in the rotating frame and rise almost parallel to the rotation axis in fast rotators. The larger the fractional depth of the convection zone, the higher the emergence latitudes, because the latitude difference between the initial and final latitudes of the rising flux tube is larger.

The second model simulates a K1IV-type fast rotator, with the mass, radius, and the rotational period of the active subgiant component of the close binary system V711 Tau (=HR 1099). The deep-seated dynamo pattern and the surface emergence are highly separated in latitude, owing to rapid rotation and convection zone geometry, as explained above. The variation of the total unsigned magnetic field for 100 years is shown in Fig. 2 . The random component in the model, ie., in latitudes and areas of BMRs at emergence, become significant for the polar regions, where the timescales for differential rotation, meridional flow, and the turbulent magnetic diffusion are too long to have an effect on the rapidly changing magnetic flux pattern. The ineffective surface transport and the highly confined, highly frequent BMR emergence thus lead to cycle-to-cycle (and long-term) fluctuations in total magnetic flux. Preliminary time-frequency analysis (Oláh 2011) indicates an interesting correspondence between the models and the long-term photometric observations of V711 Tau, as shown in Fig. 3. The dynamo cycle period in the model is about 4 years and the short-term cycle of V711 Tau is about 5 years. A longer-term 'cycle' shifts its period between 10-20 years in the model, whereas for V711 Tau a similar shifting cycle is observed between 9-18 years. A longer-term simulation for the K1IV model (Fig. 2) shows similar periods. However, it should be noted that (a) the average brightness on the one hand is compared with the total magnetic flux on the other, and (b) the relative amplitudes of the short- and long-term cycles are not similar in both cases.

\section{Discussion}

It is clear that the relationships between stellar dynamo mechanisms, emergence patterns, and surface transport processes are non-trivial. The effect of rapid rotation on rising magnetic flux tubes in stellar convection zones can lead to large departures of observable patterns from the deep-seated fields. Surface flux transport processes cause additional complications and indicate various possibilities when interpreting the observed activity patterns. Apart from several unknowns about active cool stars, we conjecture that three parameters are likely to have important effects in shaping stellar cycles: the cycle frequency, the range of latitudes of BMR emergence, and the tilt angles. 

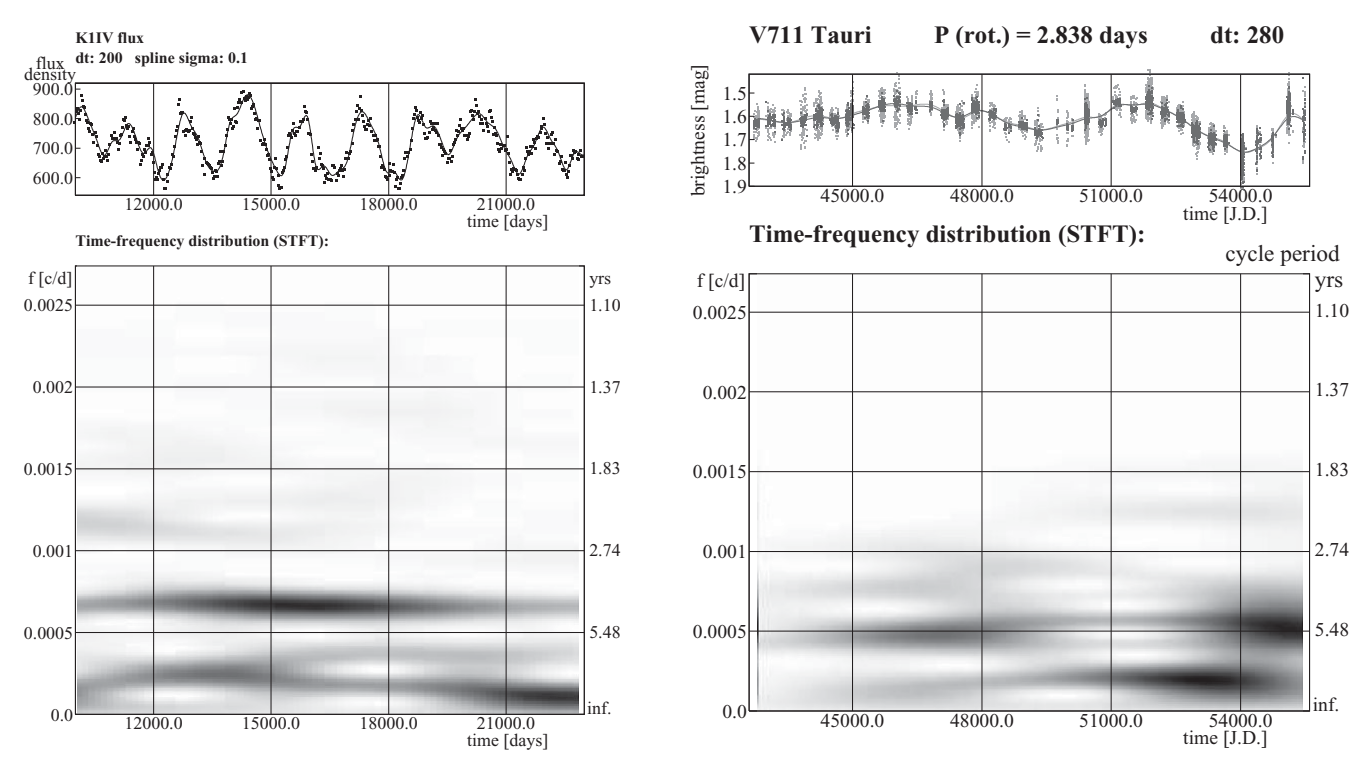

Figure 3. Time-frequency maps of the theoretical model of K1IV star with $P_{\text {rot }}=2.8 \mathrm{~d}$ (left panel, based on total unsigned magnetic flux) and V711 Tau (right panel, based on stellar brightness). For V711 Tau, the data are from Oláh et al. (2009) extended to 2011 by new measurements from the Vienna APT (Strassmeier et al. 1997). For a colour figure, see the online version of the paper.

Multiple cycles observed in active stars (Oláh et al. 2009), such as V711 Tau in Fig. 3 (right panel), do not necessarily owe their existence to periodic events taking place in the stellar interior. In the theoretical model, the short-term cycle $(\sim 4 \mathrm{yrs})$ is simply determined by the mono-periodic dynamo in the interior. The long-term cycle signal in Fig. 3 (left panel) is caused by the stochastic nature of flux emergence. Although there are several degrees of freedom in modelling magnetic fields in stellar interiors, parallel investigation of nonlinear flux-transport dynamo models and observational results will certainly be useful when estimating the relevant physical mechanisms.

\section{Acknowledgement}

The author is grateful to Katalin Oláh for the data, time-frequency analysis and useful discussions.

\section{References}

Hall, J. C. \& Lockwood, G. W. 2004, ApJ, 614, 942

Işık, E., Schmitt, D., \& Schüssler, M. 2007, $A N, 328,1111$

Işık, E., Schmitt, D., \& Schüssler, M. 2011, A\& $A$, 528, A135

Oláh, K., Kolláth, Z., Granzer, T., Strassmeier, K. G., \& Lanza, A. F. et al. 2009, AछA, 501, 703

Oláh, K. 2011, private communication

Saar, S. H. 2010, The Physics of Sun and Star Spots, IAU Symposium 273, p. 61

Schmitt, D. \& Schüssler, M. 1989, A\& A, 223, 343

Strassmeier, K. G., Boyd, L. J., Epand, D. H., \& Granzer, T. 1997, PASP, 109, 697

Strassmeier, K. G. 2009, A\&AR, 17, 251 


\section{Discussion}

Arnab Choudhuri: You have shown some results which are non-axisymmetric. How do you get them?

EMRE ISIK: The mean-field dynamo model in the tachocline is axisymmetric. However, in order to implement the two-dimensional surface flux transport, I determine longitudes of emerging bipoles randomly. 